PROPOSAL 762

Page 1-2

CASCADE SHOWERS ORIGINATED IN PROTON-NUCLEUS COLLISIONS

S. Dake, K. Nakata and H. Oda

Department of Physics, Kobe University, Kobe

T. Ogata, T. Saito and T. Tabuki

Institute for Cosmic Ray Research, University of Tokyo

T. Tominaga

Faculty of Applied Mathematics, Osaka University, Osaka

M. Fuki

Department of Physics, Okayama University of Science, Okayama

T. Shibata

Department of Physics, Aoyama Gakuin University, Tokyo

underline ... spokesman

\title{
ABSTRACT
}

Cascade showers originated in nuclear showers occurred by the proton beam with the highest energy in an emulsion chamber will be investigated for their longitudinal development and lateral structure. The results will be compared with that of $400 \mathrm{GeV}$ proton ( \#434) and that of $300 \mathrm{GeV}$ negative pions (\#506).

\section{THE PURPOSE OF THE EXPERIMENT}

The cascade showers originated in nuclear shower have been studying by using data of the emulsion chamber exposed to $400 \mathrm{GeV}$ proton beam ( \#434) and $300 \mathrm{GeV}$ negative pion beam ( \#506). The purpose of these experiment was to observe the longitudinal development and the lateral structure of the nuclear cascade shower, and the difference of their interactions.

We propose to perform the same kind of experiment as the above by exposing emulsion chambers to the proton beam of Tevatron ( $800 \mathrm{GeV}$ ). We can investigate the difference of interactions due to their energies through the evidence of these nuclear cascade showers. Especially, these inelasticity distributions to photons in previous energies could not be presented with good accuracy for the bias of low energy side, but this point in the experiment will become better by using data from the proton beam of Tevatron. 
PROPOSAL

\section{CONDITIONS REQUIRED IN THIS EXPERIMENT}

1) BEAM : parallel and mono-energetic proton beam.

2) ENERGY : $800 \mathrm{GeV}$ or maximum available energy.

3) AMOUNT OF IRRADIATION : $1.0 \times 10^{3}$ proton $/ \mathrm{cm}^{2}$ within accuracy $30 \%$.

\section{DETECTOR}

The chamber is composed of nuclear emulsion plates and lead plates which are piled up alternatively. The schematic view of the detector is shown in the figure. The geometrical size of the detector is $11.6 \mathrm{~cm} \times 11.6 \mathrm{~cm} \times 10.8 \mathrm{~cm}$ and the total thickness of lead absorber is 10-13 radiation lengths. Four chambers are planed to be exposed.

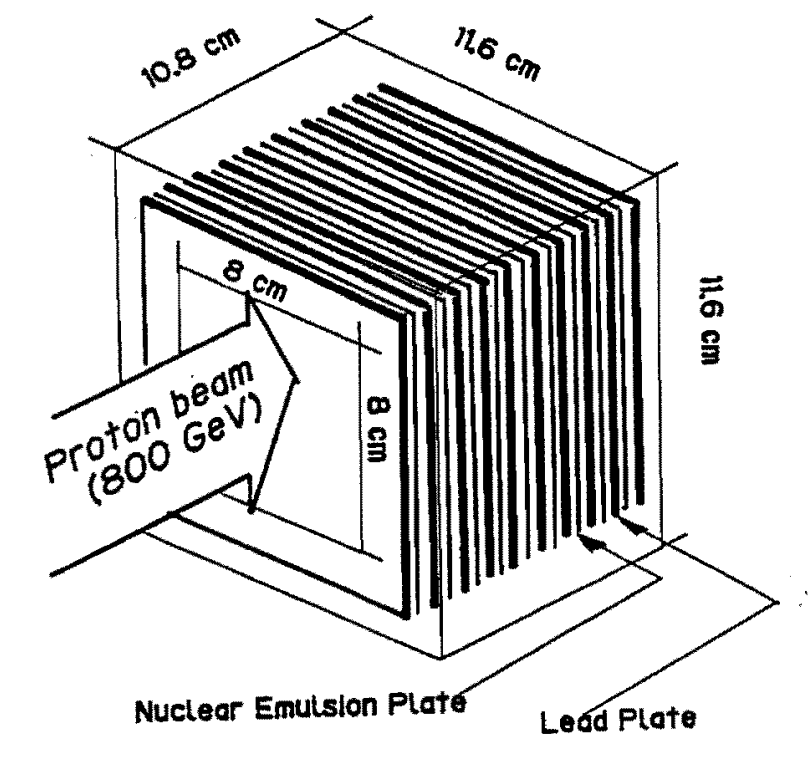

Figure. Schematic view of the chamber.

\section{REFERENCE}

1) S. Dake, et al., 14th Intern. Conf. on Cosmic Rays, Munchen, $6,2670(1975)$.

2) J. Whitmore, Phys. Report 10, 273(1974);

A. Sheng, et a1., Phys. Rev. D11, 1733(1974) 